\title{
FIGURAÇÕES IDENTITÁRIAS E CULTURAIS DO BRASIL EM LETRAS DE CANÇÕES SOB A PERSPECTIVA DE FALANTES DE OUTRAS LÍNGUAS
}

\author{
Lúcia Maria de Assunção Barbosa
}

\begin{abstract}
RESUMO: Situado no contexto de ensino e aprendizagem do português como língua estrangeira, na sua versão brasileira, este artigo propõe uma aproximação entre as dimensões linguística e cultural, vendo-as como fatores indissociáveis no processo de ensino-aprendizagem de língua estrangeira que requerem, assim, encaminhamentos metodológicos com vistas a práticas pedagógicas mais amplas. Apoiando-se no conceito de lexicultura, o estudo utiliza um corpus de unidades lexicais com cargas culturais partilhadas, retiradas de textos constituídos por letras de canções de Chico Buarque de Hollanda, vendo-as como elemento mediador de aspectos linguísticos e culturais representativos no Brasil. A partir de uma pesquisa exploratória, envolvendo um grupo de falantes de outras línguas, em situação de imersão no Brasil, a pesquisa destaca, com base nos dados obtidos, dois eixos interpretativos: um relativo às visões desse grupo referentes a elementos culturais e identitários do Brasil e dos brasileiros e outro referente ao reconhecimento de dimensões culturais (transparentes ou opacas) presentes no léxico de textos selecionados.
\end{abstract}

PALAVRAS-CHAVE: Língua estrangeira. Português língua estrangeira. Léxico. Cultura. Lexicultura.

ABSTRACT: Set in the context of learning and teaching Brazilian Portuguese as a foreign language, the aim of this article is to bring together linguistic and cultural aspects, seen as inseparable factors in the learning-teaching process of a foreign language thus requiring methodological considerations aiming at broader educational practice. Based on the lexiculture concept, the study uses a corpus of lexical units with shared cultural weight, and seen as a representative mediator of linguistic and cultural aspects in Brazil, taken from texts consisting of lyrics from songs by Chico Buarque de Hollanda. The study focuses on an experimental study involving a group of speakers of other languages in an immersion situation in Brazil. Based on data obtained from this study, the article highlights two main interpretations: one related to the views of this group concerning the cultural and identity aspects of Brazil (and of the Brazilians), and the other concerning the recognition of cultural dimensions (explicit or not) present in the lexis of the selected texts.

KEYWORDS: Foreign language. Brazilian Portuguese as a foreign language. Culture. Lexiculture.

No âmbito do ensino-aprendizagem de uma língua estrangeira, os elementos culturais têm se limitado, em geral, à exploração do potencial puramente linguístico dos conteúdos, sem a preocupação de inserir reflexões sobre os aspectos da vida cotidiana que essa língua veicula. Sabemos que uma compreensão mútua entre diferentes culturas 
requer uma visão interdisciplinar do ensino-aprendizagem, conforme preconiza a abordagem comunicativa. Associada a esta perspectiva, a noção de interculturalidade emerge como um critério indispensável para elucidar as relações entre a cultura maternal do aprendente e aquela a que ele deseja aceder, uma vez que sabemos que a linguagem é um fenômeno social e reflexo de nossa maneira de ser e de aparecer no mundo (BARBOSA, 2008).

Pretendemos mostrar, neste artigo, os resultados obtidos em um estudo qualitativo e de caráter exploratório, envolvendo um grupo de 24 falantes de outras línguas e de diferentes nacionalidades (russos, ucranianos, franceses, ingleses e latinoamericanos) que, por diferentes razões, estão no Brasil há um certo tempo. Partindo da hipótese de que formas intensivas de exposição (dos falantes de outras línguas) a situações e a textos autênticos possibilitam-lhes o acesso a um acervo lexical qualitativamente importante, objetivamos:

a) Verificar se, em textos de canções brasileiras, falantes de outras línguas identificam características culturais e identitárias atribuídas ao Brasil e a brasileiros.

b) Analisar como falantes de outras línguas interpretam o significado de palavras e expressões culturais mediadas por esses textos.

Apoiamo-nos no conceito de lexicultura e também no de palavras com cargas culturais compartilhadas, ambos propostos por Robert Galisson (1987 e 1991). De acordo com o autor, essas palavras são conhecidas e utilizadas por falantes nativos, no seu cotidiano, porém seu sentido implícito nem sempre é evidenciado para falantes de outras línguas.

A lexicultura privilegia a consubstancialidade do léxico e da cultura, mostrando o valor que as palavras adquirem pelo uso que se faz delas. Entretanto, esse valor nem sempre está presente nos dicionários. Assim, trata-se do estudo da cultura no interior do discurso, com uma vocação orientada mais pela prática e menos pela teoria e que integra, na sua concepção, a cultura no e pelo léxico, colocando-a ao alcance do aprendente de uma língua estrangeira.

A carga cultural compartilhada é o produto de julgamentos veiculados por [a] locuções figuradas, [b] locuções figuradas associando animais a defeitos ou qualidades humanas, [c] inanimados culturais (objetos fabricados ou elementos da natureza aos quais são creditadas cargas que qualquer nativo reconhece ao contato - visual ou 
auditivo - com a palavra que se refere ao objeto ou elemento). De acordo com Galisson (1997), essa carga compartilhada também resulta da [d] associação automática de um lugar a um produto, [e] do costume, do comportamento, de crenças e superstições evocadas pela palavra. No que se refere a nomes de marcas de produtos como evocadores de um conteúdo segundo, o autor ressalta que eles deslocam-se do seu objetivo primeiro (vender produtos) e passam a ter um papel importante na memória coletiva, podendo tornar-se fenômenos culturais (op. cit: 59), pois participam da composição da vida cotidiana (trivial) dos falantes nativos.

Portanto, para que esse evidencie uma carga cultural compartilhada de uma palavra, não basta apenas creditar à palavra um conteúdo cultural, pois aquilo que costumamos chamar de sentido figurado (por exemplo, a metáfora), por oposição ao sentido próprio, é produto do cultural, por estar fundamentado em analogias das formas referidas, a partir da subjetividade de um locutor coletivo, sobretudo se pensarmos que esse locutor poderia ter utilizado outra palavra para denominar o mesmo objeto.

Para este estudo, elegemos três letras de canções brasileiras, de composição de Chico Buarque de Hollanda, e procuramos estabelecer ligações entre possíveis figurações culturais e identitárias brasileiras atribuídas ao Brasil e a brasileiros e o uso efetivo da língua com base no léxico culturalmente marcado, retirado desse corpus. Privilegiamos textos de canções, em virtude da forte presença que a música tem no cotidiano da sociedade brasileira. Em função dessa inserção, ela é inegavelmente portadora de elementos culturais compartilhados pelo conjunto da coletividade e mostra-se, desse modo, como elemento pertinente para o acesso à língua e à(s) cultura(s) do país.

Para a seleção dos participantes, adotamos os critérios da amostra intencional, técnica utilizada quando o grupo é selecionado a partir de determinadas características consideradas relevantes e adequadas pelo pesquisador, para a obtenção de dados de natureza quantitativa ou qualitativa.

Desse modo, foi selecionado um grupo de 24 participantes com as seguintes características pré-estabelecidas:

a) ser adulto;

b) ser estrangeiro (falante de outra língua-cultura); 
c) estar em situação de imersão, no Brasil, num período mínimo de 1 ano e máximo de 10 anos.

Por entendermos que as particularidades e diferenças dos participantes da pesquisa (língua, idade, tempo de permanência no Brasil) podem interferir na compreensão de algumas palavras e expressões culturalmente marcadas, veiculadas nos textos de canções escolhidas, sintetizamos, no gráfico a seguir, as características relacionadas à origem do grupo:

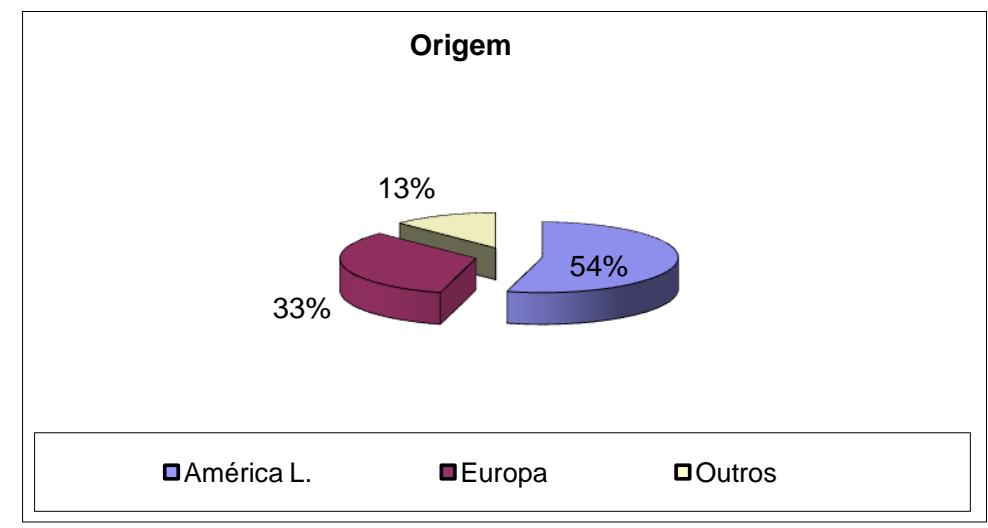

Gráfico 1: Origem

De acordo com o gráfico, vemos que a maioria dos participantes (54\%) provém de países de língua espanhola da América Latina: Argentina, Uruguai, Peru e Colômbia. Esse percentual deve-se ao fato de que esse é o grupo predominante no contexto da pesquisa. Em segundo lugar aparecem os de origem européia - franceses, ingleses, russos e ucranianos. Classificamos como outros, os países Argélia, Índia e Nova Zelândia que tiveram, cada um, um representante e que não podem ser enquadrados nas outras duas categorias. Pela representatividade de latino-americanos, a língua estrangeira predominante entre os participantes da pesquisa foi o espanhol.

No que concerne à faixa etária, dos participantes, os variava, entre 25 e 50 anos, prevalecendo um número maior de sujeitos com idades entre 25 e 39 anos.

Para coletar os dados que nos interessavam, utilizamos a técnica do questionário, a partir de um conjunto de perguntas que foram respondidas por escrito. Essa técnica mostrou-se bastante útil para a obtenção de informações acerca dos dados pessoais de cada um e também das interpretações das letras de canções. 
O questionário foi dividido em duas partes: uma referente aos itens demográficos dos participantes e outra parte contendo questões relativas à canção selecionada para ser enfocada.

A parte concernente aos dados demográficos, composta por perguntas fechadas, contém questões que possibilitaram estabelecer as aproximações necessárias entre o objeto da pesquisa e as determinações sociais ou variáveis como idade, nacionalidade, língua materna, profissão, tempo de residência no país e conhecimento de outras línguas.

A segunda parte do questionário centrou-se sobre o objeto da pesquisa propriamente dito (interpretação do texto) por isso continha questões mistas (abertas e fechadas), embora a ênfase tenha privilegiado as questões abertas por entendermos que podem fornecer-nos maiores perspectivas no momento da análise e da interpretação dos dados obtidos.

No que concerne ao primeiro objetivo - verificar se, em textos de canções brasileiras, falantes de outras línguas identificam características culturais $e$ identitárias do Brasil e dos brasileiros - priorizamos a abordagem qualitativa porque ela permite-nos interpretar as respostas e estabelecer relações entre elas. No que se refere ao segundo objetivo - analisar como falantes de outras línguas interpretam o significado de palavras e expressões culturais mediadas por canções brasileiras - não prescindimos da abordagem qualitativa, mas apresentaremos aqui apenas os dados referentes à análise quantitativa para explicitar eventuais regularidades e diferenças que os dados possam traduzir.

A interpretação e a análise dos dados obtidos foram efetuadas procurando estabelecer-se uma ligação entre as discussões teóricas previamente elaboradas e os dados da realidade pesquisada.

A partir dos dados obtidos, dividimos nossa análise em dois eixos interpretativos: um relativo às visões desse grupo referentes a elementos culturais e identitários do Brasil e dos brasileiros e outro referente ao reconhecimento de dimensões culturais (transparentes ou opacas) presentes no léxico de textos selecionados.

Entendemos que as respostas referentes às características do Brasil e dos brasileiros, apontadas pelos participantes da pesquisa enquadram-se no nível de um 
conhecimento mais observável ou factual. Ou seja, essas respostas referem-se a aspectos que hipoteticamente os participantes (por estarem em situação de imersão) podem ou puderam observar no Brasil e nos brasileiros. Esses aspectos podem ser considerados como representativos e o texto da canção pode ser um mediador dos mesmos. Além disso, esse tipo de conhecimento pode ser reportado por eles com o repertório de língua do qual eles dispõem, não sendo necessário elaborar frases longas ou mais complexas.

Por outro lado, no que se refere à questão relativa às interpretações das palavras e das expressões marcadas no texto, no nosso entendimento, essas exigem dos participantes não apenas um conhecimento, mas um reconhecimento de aspectos (e dos fatos) da cultura que estão em dimensão velada e implícita (no léxico) porque são partilhados pelos usuários (falantes nativos). Ou seja, trata-se de níveis de conhecimento diferentes: o primeiro - conhecimento - é de natureza mais observável e o segundo reconhecimento -de caráter mais interpretativo e, portanto, mais aprofundado.

Nesse sentido, é possível mostrar que aquilo que caracteriza a lexicultura reside no nível dos implícitos que perpassam a palavra e revelam práticas sociais que os falantes nativos compartilham de forma inconsciente.

Ressalte-se que as respostas dadas às letras de canções sofreram significativa influência do conteúdo textual apresentado, a começar pelo título da canção, índice que certamente interferiu na compreensão do texto.

Desse modo, obtivemos:

1- Visões exógenas do Brasil e dos brasileiros: um conhecimento para além de estereótipos culturais

Os dados da pesquisa efetuada evidenciaram que, de um modo geral, o grupo de participantes deste estudo tem uma impressão positiva em relação ao Brasil e aos brasileiros. Apesar de haver, inicialmente, uma tendência a caracterizar o país por meio de estereótipos, constatamos que esses foram imediatamente suplantados por uma visão mais crítica do país e da sociedade.

Desse modo, nas respostas atribuídas às características do Brasil e às características dos brasileiros, pudemos notar a percepção dos participantes de que o samba, o futebol e o carnaval são aspectos sociais importantes no país, mas que há outros componentes que ultrapassam essa trilogia sociocultural brasileira. Em oposição a esses três aspectos, os participantes indicaram outros pontos como: a injustiça social, o 
desemprego e os trabalhos informais e mal pagos. Essas características desmontam as imagens do tipo cartão postal, comumente exportadas através de guias turísticos, mas ainda presentes em materiais didáticos destinados ao ensino-aprendizagem de português como língua estrangeira.

Outro ponto que foi possível verificar neste estudo refere-se ao fato de que só é possível interpretar as figurações identitárias e culturais do Brasil sob a perspectiva da chamada relatividade, própria a este gênero de reflexão. Essa premissa pode ser comprovada pelas respostas dos participantes, segundo as quais o Brasil e os brasileiros não constituem uma unidade, mas sim espaços geográficos e grupos que se compõem e se opõem internamente de forma peculiar e muitas vezes incompreensível. Contudo o país e a sociedade possuem, ainda assim, traços identitários que os diferenciam de outros grupos culturais e linguísticos.

Os resultados mostram que para esses interpretantes - exógenos e em situação de imersão - o chamado ethos ou o estilo brasileiro possui como marcas as injustiças sociais evidenciadas nas condições precárias de vida, no subemprego e na violência. Nesse contexto, o jeitinho, visto por alguns como um estereótipo, pode funcionar como um elemento utilizado para vencer as dificuldades do cotidiano.

Ficou evidenciado também nesta pesquisa que os textos das canções inscrevem significações coletivas do Brasil. Por meio desses textos, o país e a sociedade revelamse de forma paradoxal, fragmentada e muitas vezes enigmática. Entretanto, ainda assim, foi possível observar, nas respostas dos participantes do estudo, a presença de um fio condutor que permite o conhecimento de uma e de outra realidade: i) a do samba, da alegria e do futebol e ii) a das dificuldades e dos paradoxos: riso/choro, trabalho/festa, comida/fome.

No que concerne às variáveis língua materna e tempo de imersão, os dados que obtivemos não nos permitiram determinar interferências das mesmas, uma vez que a competência cultural do grupo dos participantes desta pesquisa diluiu-se independentemente dessas duas variáveis. A pesquisa mostrou que, para a maioria dos participantes, os textos de canções funcionaram como mediadores de diferentes faces do país.

2. As dimensões ocultas do léxico culturalmente marcado: o reconhecimento 
Dos três textos, solicitamos que cada um participante interpretasse 14 unidades lexicais culturalmente marcadas, as quais estavam destacadas em negrito, dentro do enunciado, conforme se vê: Eles vão com uma sede de anteontem; Salta cerveja estupidamente gelada; Basta ver um rabo de saia; Pro bobo se derreter; É pirueta pra cavar o ganha-pão; Mas o que eu quero lhe dizer que a coisa aqui tá preta; Se eu ganhar algum vendendo mate; Vivo de biscate e queres que eu te sustente; Fui pegar uma cor na praia; Diz que tá dura, pendura a fatura no nosso irmão; E vamos botar água no feijão; E a gente vai se amando que, também, sem um carinho/Ninguém segura esse rojão; E a gente tá engolindo cada sapo no caminho.

De acordo com a interpretação feita por cada um, obtivemos os resultados que expomos nos gráficos 2 e 3. Para elaboração desses gráficos, foram consideradas transparentes as unidades lexicais que obtiveram respostas adequadas da metade dos participantes mais um $(50 \%+1)$. Ou seja, são transparentes as unidades lexicais que obtiveram, no mínimo, treze (13) interpretações adequadas por parte dos sujeitos da pesquisa (de um total de 24).

Considerando-se a porcentagem referida, os dados apontaram que a expressão estar (ficar) preta foi interpretada de forma adequada por $87,5 \%$ dos participantes, o que faz com que ela seja a expressão mais transparente desta pesquisa. Por outro lado, a unidade lexical saltar foi a que obteve o percentual de reconhecimento mais baixo (54\%) dentro dessa categoria (transparente).

O gráfico a seguir sintetiza os resultados referentes ao conjunto palavras e expressões consideradas transparentes. 


\section{Distribuição das expressões de acordo com nível de transparência}

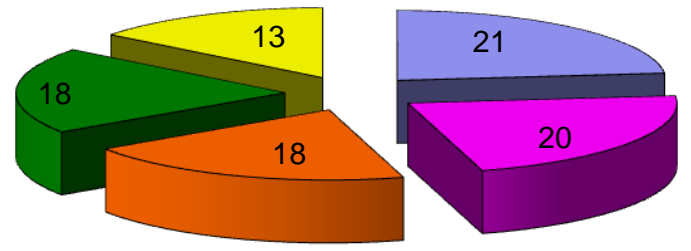

口Estar (ficar) preta

口Pegar uma cor

口Ganhar algum

-Eles vão com uma sede de anteontem

口Saltar

Gráfico 2 - Distribuição das expressões de acordo com o nível de transparência

O gráfico 2 mostra que 5 (cinco) das catorze (14) unidades lexicais apresentadas aos participantes da pesquisa obtiveram uma interpretação adequada por mais da metade dos sujeitos da pesquisa.

Os resultados também indicam que os sentidos figurados convencionais, que podemos chamar também de cristalizados, foram mais facilmente identificados, como é o caso das expressões ganhar algum e pegar uma cor, que obtiveram, respectivamente, 20 e 18 respostas consideradas pertinentes.

Outro fator que, no nosso entendimento, facilitou as interpretações de algumas unidades lexicais foi o próprio contexto discursivo. Os dados mostram que esse é o caso, por exemplo, das expressões sede de anteontem e ganhar algum. Para a unidade lexical saltar, constatamos que a semelhança com soltar funcionou como elemento favorável à compreensão do sentido.

Vale destacar ainda que essas expressões mostraram-se transparentes não apenas para falantes de línguas próximas (como o espanhol), mas pelo conjunto dos participantes. O grupo chegou ao mesmo resultado independentemente da variável lingua materna. 
A seguir, apresentamos os resultados relativos ao conjunto de unidades lexicais mais opacas, segundo a interpretação feita pelos participantes do estudo.

O gráfico 3, mostra a distribuição das expressões consideradas opacas, com a indicação em cada uma delas do número de respostas adequadas obtidas.

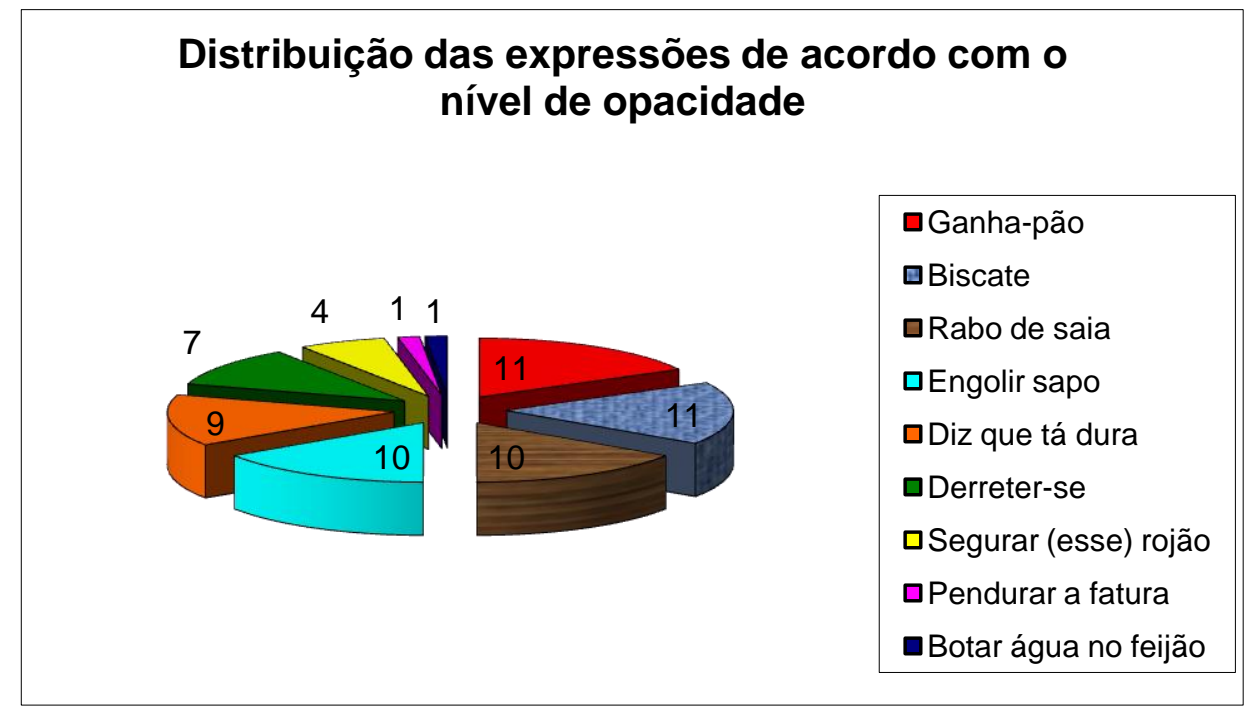

Gráfico 3 - Distribuição das expressões de acordo com o nível de opacidade

Vemos no gráfico 3 que as duas unidades lexicais que obtiveram o menor índice de reconhecimento dos participantes foram botar água no feijão e pendurar a fatura.

Para a primeira, de acordo com as respostas dos sujeitos de pesquisa, constatamos que o sentido cultural que a palavra feijão comporta não foi compreendida e que isso teve como efeito uma compreensão contrária àquela do contexto. Para o reconhecimento do sentido cultural evocado por pendurar a fatura, seria necessário que os participantes da pesquisa conhecessem o costume veiculado pelo verbo pendurar, tal como ele é utilizado no texto. Fenômeno semelhante foi produzido com a interpretação da palavra derreter-se, cuja compreensão requer dos informantes um conhecimento prévio de um certo comportamento (maneira de falar e de olhar, por exemplo) em relação à pessoa na qual se está interessado, nesse caso, uma mulher (rabo de saia).

Entretanto, a opacidade de outras expressões não ficou somente ligada ao reconhecimento ou não do sentido cultural, mas também aos elementos do texto. No caso em que a estrutura gramatical mostrou-se complexa, os resultados mostraram que isso gerou interferências na interpretação. 
No que se refere a possíveis interferências das variáveis língua materna e tempo de residência no Brasil, os dados analisados não nos permitem detectar elementos significativos em relação a essas categorias.

Desse modo, a hipótese de que falantes de línguas tipologicamente próximas como o espanhol - teriam mais facilidade para identificar, compreender e interpretar as significações culturais relativas às expressões escolhidas, não se confirma no quadro desta pesquisa, pois os dados mostram que os hispanofalantes, nesta pesquisa, obtiveram resultados semelhantes aos de falantes de outras línguas.

A análise que efetuamos dos dados coletados mostrou-nos também que a competência léxico-cultural do grupo é variável e não nos permite afirmar que essa competência seja proporcional ao tempo de imersão ou de residência no Brasil.

O que este estudo - de caráter exploratório - indica é que a interpretação preferencial, adequada ou pertinente de uma unidade lexical está ligada à compreensão mais ampla do enunciado discursivo no qual essa unidade está inserida. Em outras palavras, as significações culturais de que são portadoras certas palavras ou expressões exigem não apenas o conhecimento de seu sentido referencial, mas também um reconhecimento de outros elementos da cultura da qual o aprendente quer fazer parte.

O que se pode concluir, a partir dados obtidos, é que os 24 (vinte e quatro) participantes da pesquisa demonstraram ter uma compreensão alargada do país e de seus habitantes. Ao apontarem características do Brasil e dos brasileiros nas três letras de canções analisadas, evitaram legitimar os estereótipos culturais comumente encontrados.

Os textos de canções podem se considerados mediadores eficazes de representações do Brasil, confirmando nossa hipótese inicial de que eles exigem uma abordagem reflexiva dos elementos da cultura e contribuem para uma visão nãohomogênea de aspectos identitários e culturais do país.

Os dados permitem-nos afirmar também que aspectos mais gerais do cotidiano exigem da parte de falantes de outras línguas um conhecimento, ao passo que a interpretação de elementos implícitos, relacionados ao léxico culturalmente marcado, constitui um campo menos transparente. Do mesmo modo, fatores ligados ao texto (como a progressão temática e a estrutura sintática) podem gerar incompreensões e dificultar as interpretações esperadas. 
Nessa perspectiva, a lexicultura constitui-se como um conceito legítimo para o ensino-aprendizagem das dimensões linguístico-cultural de uma língua estrangeira, no qual o que está em jogo não é uma descrição de fatos, de vocabulário ou de gestos, mas a emergência de uma língua-cultura que decifra, analisa, descobre e interpreta práticas sociais implícitas nas interações cotidianas.

\section{REFERÊNCIAS}

BARBOSA, Lúcia Maria de Assunção. Opacité et transparence lexico-culturelle dans l'apprentissage du portugais langue etrangère au Brésil: les paroles de chansons, instruments de médiation linguistique et culturelle. Lille: Atellier National de Reproduction de Thèses, 2008.

GALISSON, Robert. Accéder à la culture partagée par l'entremise des mots à CCP . Études de Linguistique Apliquée, Paris, nº 67, 109-15, 1987.

GALISSON, Robert. De la langue à la culture par les mots. Paris : CLE International, 1991.

Un dictionnaire à géométrie variable au service de la lexiculture. Cahiers de lexicologie, Paris, no 70/1, 57-77, 1997.

RECEBIDO EM: 01 de novembro de 2012

APROVADO EM: 14 de dezembro de 2012 